\title{
Velvet Antler Methanol Extracts Ameliorate Parkinson's Disease by Inhibiting Oxidative Stress and Neuroinflammation: From C. elegans to Mice
}

\author{
Ying Liu, ${ }^{1}$ Hongyuan $\mathrm{Li}^{2,3}$ Yunfei $\mathrm{Li}^{4}{ }^{4}$ Min Yang, ${ }^{1}$ Xiaohui Wang $\mathbb{D}^{2,5}$ and Yinghua Peng $\mathbb{D}^{1}$ \\ ${ }^{1}$ State Key Laboratory for Molecular Biology of Special Economic Animal, Institute of Special Animal and Plant Sciences, \\ Chinese Academy of Agricultural Sciences, Changchun, Jilin 130112, China \\ ${ }^{2}$ Laboratory of Chemical Biology, Changchun Institute of Applied Chemistry, Chinese Academy of Sciences, Changchun, \\ Jilin 130022, China \\ ${ }^{3}$ State Key Laboratory of Medicinal Chemical Biology, Nankai University, Tianjin 300071, China \\ ${ }^{4}$ Department of Pharmaceutical Engineering, College of Humanities \& Information, Changchun University of Technology, \\ Changchun 130122, China \\ ${ }^{5}$ Department of Applied Chemistry and Engineering, University of Science and Technology of China, Hefei 230026, China
}

Correspondence should be addressed to Xiaohui Wang; xiaohui.wang@ciac.ac.cn and Yinghua Peng; yhpengtcs@126.com

Received 21 August 2020; Revised 15 November 2020; Accepted 3 December 2020; Published 9 January 2021

Academic Editor: Gr gory Durand

Copyright ( 2021 Ying Liu et al. This is an open access article distributed under the Creative Commons Attribution License, which permits unrestricted use, distribution, and reproduction in any medium, provided the original work is properly cited.

Velvet antler is the traditional tonic food or medicine used in East Asia for treating aging-related diseases. Herein, we try to dissect the pharmacology of methanol extracts (MEs) of velvet antler on Parkinson's disease (PD). Caenorhabditis elegans studies showed that MEs decreased the aggregation of $\alpha$-synuclein and protected oxidative stress-induced DAergic neuron degeneration. In vitro cellular data indicated that MEs suppressed the LPS-induced MAPKs and NF- $\kappa \mathrm{B}$ activation, therefore inhibiting overproduction of reactive oxygen species, nitric oxide, tumor necrosis factor- $\alpha$, and interleukin-6; blocking microglia activation; and protecting DAergic neurons from the microglia-mediated neurotoxicity. In vivo MPTP-induced PD mouse investigations found that MEs prevented MPTP-induced neuron loss in the substantia nigra and improved the behavioral rotating rod performance in MPTP-treated mice by increasing the expression level of tyrosine hydroxylase (TH) and downregulating $\alpha$-synuclein protein expression. In all, these results demonstrate that MEs ameliorate PD by inhibiting oxidative stress and neuroinflammation.

\section{Introduction}

Parkinson's disease (PD) is the second most common neurodegenerative disorder after Alzheimer's disease and is still incurable [1]. It is characterized by motor symptoms such as uncontrollable tremor, muscle stiffness, and slowness of movement [2]. PD is characterized by severe degeneration of dopaminergic (DAergic) neurons in substantia nigra (SN) and depletion of dopamine in the striatum. The etiology and pathogenesis of PD so far have not been completely elucidated, although current theories suggest that oxidative stress and neuroinflammation exert DAergic neuron demise and are involved in neuronal degeneration of PD [3].

Currently, there are few therapeutic options for preventing and treating PD, which only treat symptoms and do not retard DAergic neuron degeneration. Velvet antler has been used as traditional Chinese medicine and tonic food in East Asia for thousands of years [4], which has been reported to exert anti-inflammatory and antiaging effects $[5,6]$. Methanol extracts (MEs) of velvet antler, which are rich in terpenoids, phenol, steroids, lipids, and glycosides and have no protein substances, protect against oxidative stress in 
Caenorhabditis elegans (C. elegans) [7]. Herein, we try to investigate whether MEs could ameliorate PD based on $C$. elegans and mouse models. MEs were found to inhibit reactive oxygen species (ROS) and neuroinflammation and therefore prevented the degenerations of DAergic neurons, which indicate that MEs would be an effective therapeutic agent against $\mathrm{PD}$.

\section{Materials and Methods}

2.1. Materials. Velvet antler from sika deer (Cervus nippon) was provided by the Zuojia Sika Deer Farm (Jilin, China). The preparation and composition of velvet antler methanol extracts (MEs) were previously described [7]. Simply, $7 \mathrm{~g}$ of antler velvet powder was mixed with $210 \mathrm{~mL}$ methanol and was refluxed at $80^{\circ} \mathrm{C}$ for $1 \mathrm{~h}$. The supernatant was obtained after centrifugation at $8000 \mathrm{~g}$ for $15 \mathrm{~min}$. The methanol solvent was removed under vacuum with a rotary evaporator. The yield of MEs was 3.2\% $(w / w)$ of the dried sample. 6-Hydroxydopamine (6-OHDA), 2,3-diaminonaphthalene, $2^{\prime}, 7^{\prime}$-dichlorodihydrofluorescin diacetate (DCFH-DA), crystal violet, BCA assay kit, 1-methyl-4phenyl-1,2,3,6-tetrahydropyridine (MPTP), and hematoxyli$\mathrm{n} /$ eosin were obtained from Sigma-Aldrich (St. Louis, MO, USA). Interleukin-6 (IL-6) and tumor necrosis factor $\alpha$ (TNF- $\alpha$ ) ELISA kits were purchased from Abcam (Cambridge, MA, USA). The Cell Death Detection kit was purchased from Roche Applied Science (Basel, Switzerland). The primary antibodies against IBA-1, p-ERK, p-JNK, p-p38, GAPDH, p-p65, p-Akt, tyrosine hydroxylase (TH), and $\alpha$-synuclein as well as the horseradish peroxidase- (HRP-) conjugated secondary antibody were purchased from Cell Signaling Technology (Beverly, MA, USA). Alexa Fluor 568 to goat IgG secondary antibody was purchased from Thermo Fisher Scientific (Waltham, MA, USA).

2.2. C. elegans DAergic Degeneration Measurement. A transgenic strain BZ555, which expresses GFP in DAergic neurons through the dat-1::GFP reporter system, was used to assess the effect of MEs on DAergic neurons. Adult worms were incubated for $6 \mathrm{~h}$ at $20^{\circ} \mathrm{C}$ and allowed to lay eggs; then, synchronized worms at the L4 larval stage were treated with 6OHDA. At the end of exposure, worms were spread on the NGM OP50 plates with or without MEs. Worms at days 1, 2 , and 3 past the adult stage were mounted onto $2 \%$ agarose pads and immobilized with $2 \mathrm{mM}$ levamisole and imaged with a Nikon TS2-FLfluorescence microscope. DAergic neurons were counted by inspecting the GFP fluorescence, which could be quantified with ImageJ. At least 30 worms were examined with three replicates.

2.3. C. elegans Basal Slowing Response (BSR) Assay. The C. elegans strain N2 was handled according to standard procedures and grown at $20^{\circ} \mathrm{C}$. $\mathrm{N} 2$ adult worms were incubated for $6 \mathrm{~h}$ at $20^{\circ} \mathrm{C}$ and allowed to lay eggs. The synchronized worms at the L4 larval stage were treated with M9 buffer containing $10 \mathrm{mM}$ ascorbic acid and $50 \mathrm{mM}$ 6-OHDA for $1 \mathrm{~h}$. The worms were then washed in M9 buffer and spread on NGM OP50 plates with or without MEs. Ten worms at days 1,2 , and 3 past the adult stage were collected by a washing plate with M9 buffer and then transferred to plates with or without a ring-shaped OP50 lawn. 5 min later, the number of body bending was counted to assess the locomotor rate in $20 \mathrm{~s}$ duration. Data is expressed as the difference $(\Delta)$ in body bending per $20 \mathrm{~s}$ between worms in OP50 seeded plates and plates without food, which is a measurement of 6-OHDA oxidation damage on dopaminergic neurons in C. elegans. At least three replicates were performed independently.

2.4. C. elegans $\alpha$-Synuclein Aggregation Measurement. A transgenic strain, NL5901[unc-54p::alpha synuclein::YFP +unc-119(+)], which stably expresses human alpha synuclein protein tagged with yellow fluorescent protein, was used to assess the PD in the worm. Briefly, NL5901 strain nematodes were exposed to MEs from the L4 larval stage to days 3 and 5 past the adult stage. At the end of exposure, worms were mounted onto $2 \%$ agarose pads and immobilized with $2 \mathrm{mM}$ levamisole. To monitor the $\alpha$-synuclein aggregation, YFP protein was microscopically visualized and photographed using a Nikon TS2-FLfluorescence microscope. At least 30 worms examined with three replicates were imaged, and the fluorescence signals were quantified in each worm with ImageJ software.

2.5. Cell Viability Assay. A mouse microglial BV2 cell line was cultured in Dulbecco's modified Eagle's medium (DMEM) containing $10 \%$ FBS, $4 \mathrm{mM}$ glutamine, $100 \mathrm{U} / \mathrm{mL}$ penicillin, and $100 \mu \mathrm{g} / \mathrm{mL}$ streptomycin at $37^{\circ} \mathrm{C}$ in a $5 \% \mathrm{CO}_{2}$ incubator. $\mathrm{BV} 2$ cells were exposed to different treatments as indicated. After $24 \mathrm{~h}$ treatment, cells were fixed with $3.7 \%$ paraformaldehyde for $5 \mathrm{~min}$ and then stained with $0.05 \%$ crystal violet for $15 \mathrm{~min}$. The plates were subsequently washed with tap water and dried for $30 \mathrm{~min}$ at room temperature; $200 \mu \mathrm{L}$ of methanol was added to each well, and the plates were shaken for $15 \mathrm{~min}$ at room temperature to dissolve the dye. Absorbance at $540 \mathrm{~nm}$ was measured using a plate reader.

2.6. ROS Measurement in BV2 Cells. BV2 cells were exposed to different treatments for $24 \mathrm{~h}$ as indicated. Cells were washed with PBS and then loaded with $\mathrm{H}_{2}$ DCFDA at a final concentration of $5 \mu \mathrm{M}$ in a serum-free medium. $\mathrm{H}_{2}$ DCFDA is a nonfluorescent and cell-permeable probe that is converted into $2^{\prime}, 7^{\prime}$-dichlorodihydrofluorescein after intracellular deacetylation and is subsequently oxidized to highly fluorescent dichlorofluorescein (DCF). Cells were incubated for $15 \mathrm{~min}$ and then washed. Cellular fluorescence was viewed by a Nikon TS2-FLfluorescence microscope.

2.7. NO Assay. BV2 cells were exposed to different treatments for $24 \mathrm{~h}$ as indicated. $100 \mu \mathrm{L}$ of supernatant media was removed after cells were treated for $24 \mathrm{~h}$ and added to flat black 96-well microfluor plates (Thermo Scientific, Waltham, MA, USA). Subsequently, $10 \mu \mathrm{L}$ of 2,3-diaminonaphthalene $(0.05 \mathrm{mg} / \mathrm{mL}$ in $0.62 \mathrm{M} \mathrm{HCl})$ was added to each well and incubated for $15 \mathrm{~min}$. The reaction was quenched by the addition of $5 \mu \mathrm{L}$ of $3 \mathrm{M} \mathrm{NaOH}$, and the plate was read on a plate reader with excitation at $360 \mathrm{~nm}$ and emission at $430 \mathrm{~nm}$. 
2.8. TNF- $\alpha$ and IL-6 ELISA. BV2 cells were exposed to different treatments as indicated. After $24 \mathrm{~h}$ treatment, culture supernatants were collected for TNF- $\alpha$ and IL-6 ELISAs, which were performed according to the manufacturer's instructions (Abcam, Cambridge, MA, USA). Briefly, the capture antibody was added in each well of a 96-well ELISA plate and incubated overnight at $4^{\circ} \mathrm{C}$. The plate was washed with phosphate-buffered saline (PBS) with $0.05 \%$ Tween 20 solution (PBST) five times and then incubated with $100 \mu \mathrm{L}$ supernatant/each well for $2 \mathrm{~h}$ at room temperature. Following five PBST washings, biotin-conjugated antibody was added to each well and incubated for $1 \mathrm{~h}$ at room temperature. After five PBST washings, diluted avidin-HRP was added and the plate was incubated at room temperature for $30 \mathrm{~min}$. After washing the plate five times, tetramethylbenzidine (TMB) substrate was added to each well, and the color was developed in the dark for 10-30 min at room temperature. The color reaction was stopped by adding $1 \mathrm{M} \mathrm{H}_{3} \mathrm{PO}_{4}$. The absorbance at $450 \mathrm{~nm}$ was measured on a plate reader, and $620 \mathrm{~nm}$ was chosen as the reference wavelength. The concentration of TNF- $\alpha$ and IL- 6 was calculated using a mouse TNF- $\alpha$ and IL- 6 standard working curve, respectively.

2.9. Iba-1 Immunofluorescence Staining. BV2 cells were exposed to different treatments as indicated. After $24 \mathrm{~h}$ treatment, cells were fixed with $4 \%$ paraformaldehyde. $1 \%$ bovine serum albumin (BSA) in PBS was used for blocking for $30 \mathrm{~min}$. The cells were incubated overnight at $4^{\circ} \mathrm{C}$ with Iba- 1 primary antibody diluted $1: 250$ in 1\% BSA in PBS. After five washings with PBS, the cells were incubated with a secondary Alexa Fluor 568-conjugated anti-goat IgG for $1 \mathrm{~h}$ at room temperature. The cellular nucleus was stained with $1 \mu \mathrm{g} / \mathrm{mL}$ of Hoechst 33258 for $5 \mathrm{~min}$. The staining of BV2 cells was visualized by a Nikon TS2-FLfluorescence microscope.

2.10. TUNEL Assay. BV2 cells were exposed to different treatments as indicated. After $24 \mathrm{~h}$ treatment, cells were fixed with $4 \%$ paraformaldehyde, and terminal deoxynucleotidyl transferase dUTP nick end labeling (TUNEL) staining was performed by the Cell Death Detection kit (Roche Applied Science, Basel, Switzerland) according to the manufacturer's instruction. DNA fragmentation was detected using a Nikon TS2-FLfluorescence microscope.

2.11. Western Blotting. The lysate samples were first resolved in $12 \%$ sodium dodecyl sulfate-polyacrylamide gel electrophoresis (SDS-PAGE) and then transferred to nitrocellulose membranes, followed by blocking membranes with $5 \%$ nonfat dry milk in TBST buffer ( $25 \mathrm{mM}$ Tris- $\mathrm{HCl}, 140 \mathrm{mM} \mathrm{NaCl}$, $0.05 \%$ Tween $20, \mathrm{pH} 7.5$ ) for $1 \mathrm{~h}$ and incubation with the appropriate primary antibody $(0.5 \mu \mathrm{g} / \mathrm{mL})$ at $4{ }^{\circ} \mathrm{C}$ overnight. After being washed five times by TBST, the membrane was incubated with horseradish peroxidase-conjugated secondary antibody $(50 \mathrm{ng} / \mathrm{mL})$ for $1 \mathrm{~h}$ at room temperature. After sufficient washing, antibody complexes attached to the membrane were visualized by a Tanon-5200 Multi when reacting with SuperSignal West Pico Chemiluminescent Substrate (Pierce, Rockford, IL, USA). ImageJ was used for densitometric analysis.
2.12. In Vivo Mouse Behavioral Studies. Pathogen-free adult male C57BL/6 mice weighing 20-25g were used in all experiments (Liaoning Changsheng Biotechnology, China). Mice were housed in temperature-controlled $\left(18-21^{\circ} \mathrm{C}\right)$ and light-controlled ( $12 \mathrm{~h}$ light-dark cycle; lights on at 7:00 am) rooms with standard rodent food and water available ad libitum and allowed to habituate to the holding facility for $\geq 1$ week prior to experimentation. All the animal-handling procedures were performed in strict accordance with the regulations for the Administration of Affairs Concerning Experimental Animals approved by the State Council of the People's Republic of China (11-14-1988).

The mice that pretrained on the rotating rod and had no difference in the time of staying on the rod were randomly divided into three groups (6 per group): the control group (intraperitoneal injection of a saline solution for 5 days), MPTP-treated groups (intraperitoneal injection of $30 \mathrm{mg} / \mathrm{kg}$ MPTP for 5 days), and MPTP plus ME-treated group (intraperitoneal injection of $30 \mathrm{mg} / \mathrm{kg}$ MPTP and $30 \mathrm{mg} / \mathrm{kg}$ MEs for 5 days). The animals were subjected to behavioral testing $48 \mathrm{~h}$ after the final injection and then sacrificed.

PD-induced behavioral changes were measured by the rotarod performance test as described previously [8]. In brief, mice performed three $10 \mathrm{~min}$ pretraining trials daily for 3 consecutive days before MPTP treatment. The rotarod performance test used a rod with a diameter of $3 \mathrm{~cm}$ and set at $40 \mathrm{rpm}$ rotation. Mice were placed on the rotating rod, and the time for which each mouse maintained balance on the rod was recorded. Each mouse was tested three times with an intertrial interval of $20 \mathrm{~min}$. The average time was considered the final score.

2.13. Immunohistochemistry. After the behavioral studies, brain tissues were dissected and fixed in universal tissuefixed fluid at $4^{\circ} \mathrm{C}$ for 24 and then embedded in paraffin. Immunohistochemistry (IHC) was then performed. The tissue slides $(3 \mu \mathrm{m})$ were deparaffinized with xylene and rehydrated in alcohol, washed with PBS, and incubated in $10 \mathrm{mM}$ citrate buffer ( $\mathrm{pH} \mathrm{7.4)}$ at $90^{\circ} \mathrm{C}$ for $15 \mathrm{~min}$. The slides were then treated with $0.3 \%$ hydrogen peroxide in methanol at $4^{\circ} \mathrm{C}$ for $30 \mathrm{~min}$ to inactivate endogenous peroxidases. After blocking with $5 \% \mathrm{BSA}$ for $25 \mathrm{~min}$ at $25^{\circ} \mathrm{C}$, the tissues were washed with PBS and incubated with primary antibody overnight at $4^{\circ} \mathrm{C}$. Subsequently, they were incubated with secondary antibody for $30 \mathrm{~min}$. DAB was used as the chromogenic agent, and hematoxylin was used as the dye reagent. The tissue slides were blocked and then observed under a microscope.

2.14. Immunofluorescence Staining of NF- $\kappa B$ in Substantia Nigra (SN). After the behavioral studies, brain tissues were dissected and fixed in $4 \%$ paraformaldehyde and embedded in paraffin. The brains were then cut into $3 \mu \mathrm{m}$ coronal sections with a paraffin microtome and followed by heatinduced antigen retrieval. Sections containing substantia nigra regions were subjected to immunostaining. Endogenous peroxidase activity was quenched by incubation in $1 \%$ hydrogen peroxide in methanol for $30 \mathrm{~min}$ and then cleared in PBS for $5 \mathrm{~min}$. The sections were blocked for $30 \mathrm{~min}$ with 


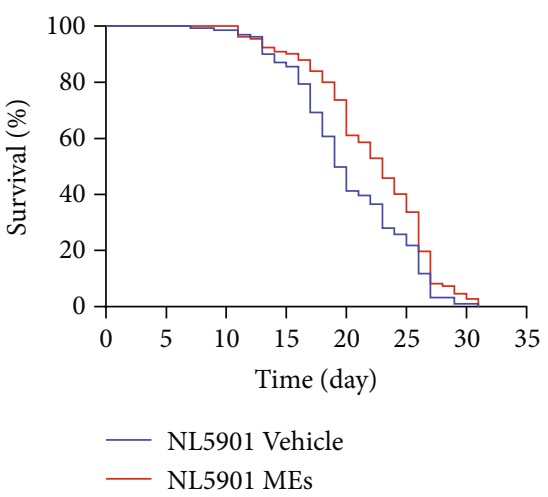

(a)

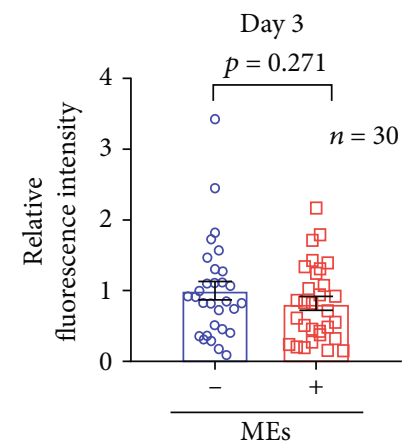

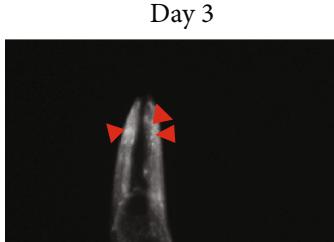

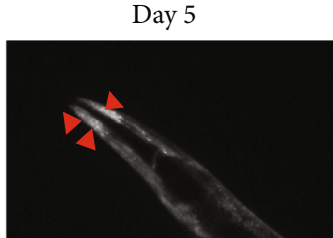

$\sum^{\infty}$
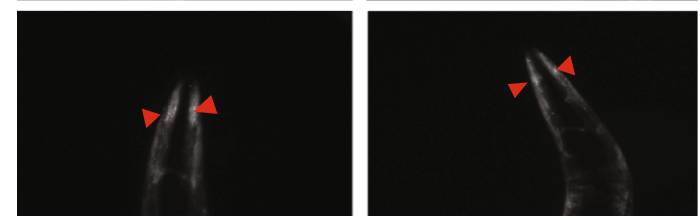

(b)

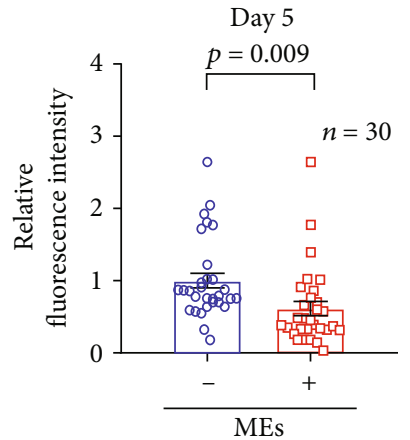

(c)

FIGURE 1: MEs decreased the aggregation and toxicity of $\alpha$-synuclein in NL5901 worms. (a) The survival curve of NL5901 worms treated with MEs. (b) The endpoint microscope fluorescence image of $\alpha$-synuclein aggregates in NL5901 worms treated with or without MEs on days 3 and 5 past the adult stage. (c) The quantitative analysis of the $\alpha$-synuclein aggregates in NL5901 worms shown in (b). All the worms in the experiment were synchronized to the young adult stage and subsequently started to be exposed to $100 \mu \mathrm{g} / \mathrm{mL} \mathrm{MEs}$. Error bars represented the SEM of three independent replicates of total worms.

bovine serum albumin diluted in PBS. These sections were incubated with primary antibody against NF- $\kappa \mathrm{B}$ protein (ab16502, Abcam, UK) overnight at $4^{\circ} \mathrm{C}$. After washing in PBS, the sections were incubated in cy3-conjugated secondary antibody (Servicebio, China) for $1 \mathrm{~h}$ at room temperature. Washing of the sections was again done three times and then incubated with DAPI solution at room temperature for 10 min. The sections were subsequently washed with PBS and viewed under a fluorescence microscope.

2.15. Hematoxylin/Eosin Staining. Tissues were fixed in universal tissue fixation fluid at $4^{\circ} \mathrm{C}$ for $24 \mathrm{~h}$ and embedded in paraffin. Tissue sections $(3 \mu \mathrm{m})$ were deparaffinized and stained with hematoxylin/eosin (H-E). At least five paraffin sections from each tissue were used for $\mathrm{H}-\mathrm{E}$ staining.

\section{Results and Discussions}

3.1. MEs Inhibit $\alpha$-Synuclein Aggregation and Protect DAergic Neurons in C. elegans. C. elegans is a good model organism for the study of pathogenesis and drug discovery for PD [9], owing to several advantages including a completely sequenced genome [10], numerous mutants and multicolor reporter constructs freely available $[11,12]$, a rapid replication cycle, and ease of growing and maintenance as well as manipulation [13]. Increasing evidence suggests that oxidative stress plays a major role in the development of PD [14]. Our recent work showed that MEs of velvet antler protected against oxidative stress in C. elegans. Therefore, whether MEs could affect the progression of PD was investigated. NL5901 strain, which has been created by inserting human $\alpha$-synuclein gene with YFP fusion construct driven by the unc- 54 promoter, was used as a PD model. As shown in Figure 1(a), ME treatment significantly prolonged the lifespan of NL5901 worms when compared to the untreated control. NL5901 worms exhibited aggregation of $\alpha$-synuclein (Figure 1(b)). The YFP intensities were quantitatively analyzed, and the mean fluorescent intensity in the control group was set as 1 . Although not statistically significant, there was a trend which suggested that ME treatment decreased the $\alpha$-synuclein-YFP intensities on day 3 (Figure 1(c)). Moreover, ME treatment significantly decreased the $\alpha$-synucleinYFP intensities on day 5 when compared to the untreated control (Figure 1(c)). These results indicate that MEs decrease 
the aggregation of $\alpha$-synuclein and therefore improve the lifespan of the worm model of PD.

$\mathrm{PD}$ is typically associated with degeneration of the DAergic neurons [15], which are particularly prone to oxidative stress [16]. Whether MEs could protect DAergic neurons was next investigated in $C$. elegans. In contrast to rodents which have 10,000-20,000 DAergic neurons, or humans which have greater than 40,000 DAergic neurons [17], C. elegans have only eight DAergic neurons: two anterior, four cephalic, and two posterior deirid neurons [18], which makes the in situ investigation of the vulnerability of DAergic neurons to oxidative stress possible. BZ555 strain, in which all DAergic neurons are tagged with GFP by fusing with DAT-1, was used to visualize the bodies of DAergic neurons. 6-Hydroxydopamine (6-OHDA), a redox cycling dopamine analog and an oxidative neurotoxin, was used to lesion dopaminergic pathways and generate an experimental model for PD [19]. BZ555 worms were treated as indicated, and the green fluorescence was analyzed in the nerve ring (Figure 2(a) and Figure S1), which contains GFP-tagged DAergic neurons. Compared to the untreated control, 6OHDA treatment caused a significant shrinkage of the soma of DAergic neurons (Figure 2(b)). ME treatment slightly increased the nerve ring of DAergic neurons under the normal condition and significantly ameliorated the 6-OHDAinduced degeneration of DAergic neurons (Figure 2(b)).

To confirm the results from the transgene BZ555 worms, the effect of MEs on the functionality of DAergic neurons under the oxidative stress was evaluated by the basal slowing response (BSR) assay in the wild-type N2 worms. This behavioral assay measures the ability of wild worms to slow down their rate of locomotion when they encounter a bacterial lawn, which is mediated by DA. As shown in Figure 2(c), 6OHDA treatment induced a reduction in BSR as compared to the untreated control. MEs increased the BSR value of $\mathrm{N} 2$ worms treated with 6-OHDA but did not affect the BSR of the wild-type worms under the normal condition (Figure 2(c)). These functional data revealed by BSR of the wild-type N2 worms are consistent with the direct DAergic neuron observations in the transgene BZ555 worms. Together, these C. elegans data demonstrate that MEs inhibit $\alpha$-synuclein aggregation and protect DAergic neurons in $C$. elegans from degeneration.

3.2. MEs Inhibit ROS and Proinflammatory Factors in the Activated Microglia. The above C. elegans data implies that MEs of velvet antler inhibit the development of PD. To test whether MEs could be a potential means for preventing and treating $\mathrm{PD}$, the effects of MEs were further tested in the mammalian system. Before moving to in vivo mouse studies, the actions of MEs were tested in the microglia, which are the main immune effector cells in the central nervous system (CNS) and play a major role in PD pathology [20]. Although a variety of potential sources for ROS exist in the CNS, the microglia generate large quantities of these reactive species [21]. Microglia BV2 cells were used herein since they reproduce many of the responses of primary microglia with high fidelity [22]. To explore the effect of MEs on the LPS-induced ROS production, the bacterial endotoxin, lipopolysaccharide (LPS), which has been the most extensively utilized microglia activator for the induction of DAergic neurodegeneration [23], was used to activate BV2 cells. Intracellular ROS generation was measured by fluorescence staining with $\mathrm{H}_{2}$ DCF-DA. No apparent green fluorescence was observed for the untreated BV2 cells (Figure 3(a)). LPS stimulation caused the ROS burst in BV2 as revealed by the strong green fluorescence from DCF (Figure 3(a)), suggesting the activation of microglia. MEs significantly inhibited the LPS-induced DCF fluorescence intensity (Figure 3(a)), which indicates that MEs reduce the oxidative stress in the activated microglia. Excess ROS directly inflicts DNA damage, and TUNEL staining was performed to assess oxidative stress by measuring DNA fragmentation. Compared to the untreated control, LPS stimulation caused DNA fragmentation in BV2 cells as reflected by TUNEL fluorescence (Figure 3(b)). MEs protected against the LPS-induced DNA fragmentation (Figure 3(b)). Together, these results from microglia are consistent with the previous observation that MEs protect against oxidative stress in C. elegans [7]. It should be noted that the effect of MEs on cellular viability was measured by crystal violet staining. No apparent cellular toxicity was observed (Figure S2), even at the concentration of $80 \mu \mathrm{g} / \mathrm{mL}$ of MEs, which eliminates the possibility that the observed ROS inhibition by MEs was due to the artifact like cell death.

In addition to ROS, accumulating evidence points to activated microglia as a main source of several proinflammatory factors, including nitric oxide (NO), tumor necrosis factor- $\alpha$ (TNF- $\alpha$ ), and interleukin-6 (IL-6), driving progressive DAergic neurodegeneration and PD development [24, 25]. The effect of MEs on the proinflammatory mediators in the activated microglia was investigated. LPS induced NO (Figure 4(a)), TNF- $\alpha$ protein (Figure 4(b)), and IL-6 protein (Figure 4(c)) overproduction in BV2 cells. MEs inhibited the LPS-induced NO (Figure 4(a)), TNF- $\alpha$ (Figure 4(b)), and IL6 (Figure $4(c)$ ) in a concentration-dependent manner. These results imply that MEs have antineuroinflammation activity.

Ionized calcium-binding adaptor molecule 1 (Iba-1) is specifically expressed in microglia/macrophages and is involved with the membrane ruffling and phagocytosis in activated microglia [26]. Iba-1 is upregulated during the activation of microglia, which is therefore used as the marker of microglia activation [27]. As shown in Figure 4(d), Iba-1 was expressed on the plasma of BV2 cells, where a green fluorescence signal was observed. LPS stimulation increased the expression of Iba-1, which was inhibited by MEs. Together, these data show that MEs inhibit microglial activation, which is consistent with the suppression of LPS-induced ROS and proinflammatory factor (NO, TNF- $\alpha$, and IL-6) overproduction by MEs.

Oxidative stress and neuroinflammation cause neuronal cell degeneration [25, 28-31]. To directly explore whether MEs could protect neuron cells from the damages caused by oxidative stress and neuroinflammation from the activated microglia, the effect of conditional media from BV2 cells on SH-SY5Y cellular viability was investigated. SH-SY5Y cells were used as an in vitro model of DAergic neurons for PD research, because they possess many characteristics of DAergic neurons [32]. The conditioned medium from the LPS-treated 


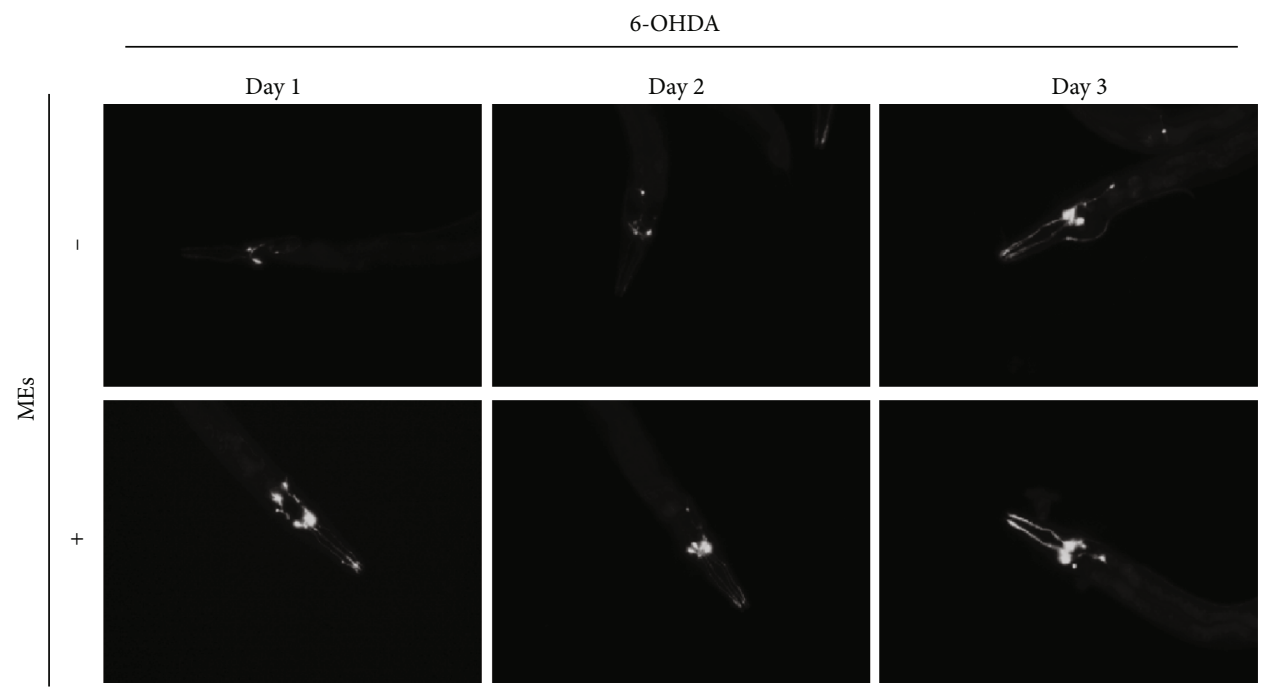

(a)

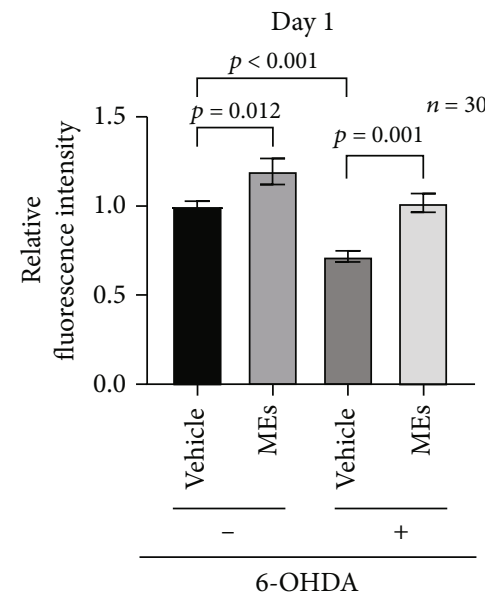

Day 1

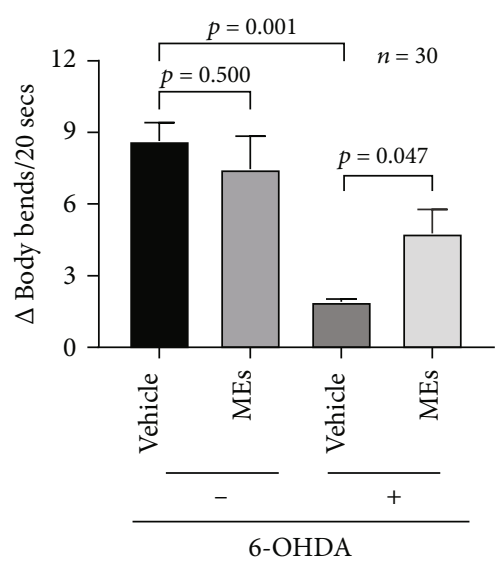

Day 2

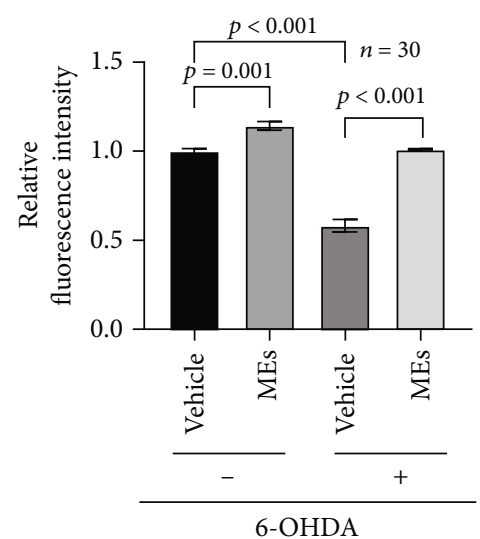

(b)

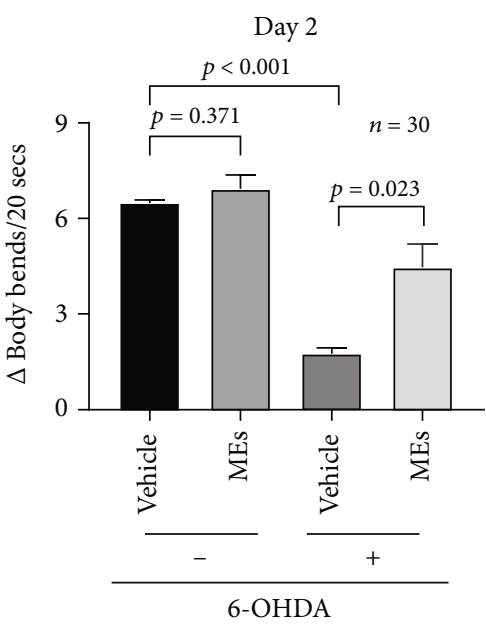

Day 3
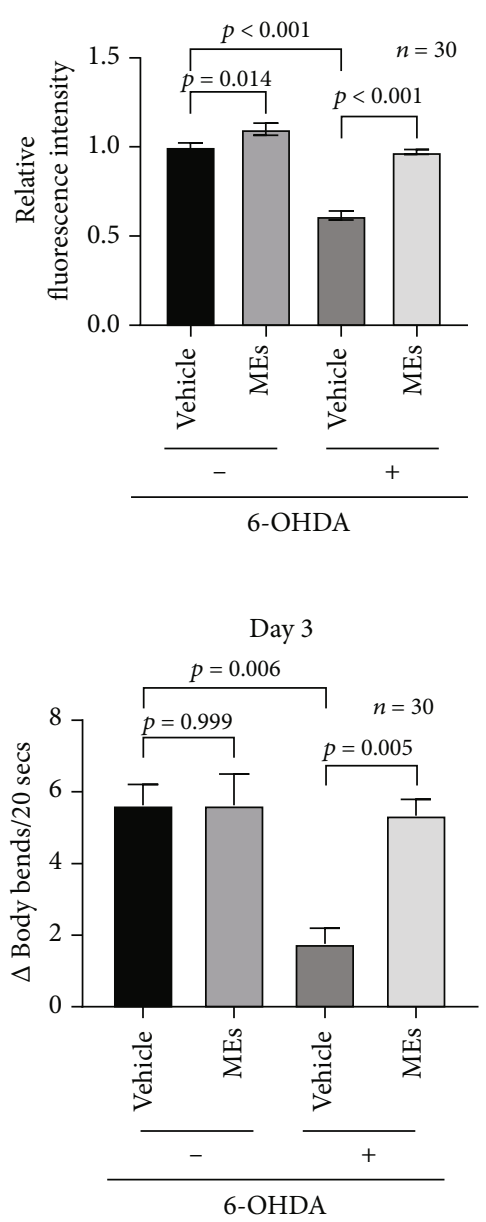

(c)

FIGURE 2: MEs protected against neuron injury induced by 6-OHDA in C. elegans. (a) The endpoint microscope fluorescence image of DAergic neurons in BZ555 worms treated with or without MEs on days 1, 2, and 3 past the adult stage. The BZ555 worms synchronized at the L4 larval stage were exposed to 6-OHDA and subsequently started to be exposed to $100 \mu \mathrm{g} / \mathrm{mL} \mathrm{MEs} \mathrm{for} \mathrm{1-3} \mathrm{days.} \mathrm{(b)} \mathrm{The}$ quantitative analysis of the intensity of DAergic neurons shown in (a). (c) The difference of the average number of body bends per $20 \mathrm{~s}$ between $\mathrm{N} 2$ worms in OP50 seeded plates and plates without food. N2 worms synchronized at the L4 larval stage were exposed to 6-OHDA and subsequently started to be exposed to $100 \mu \mathrm{g} / \mathrm{mL}$ MEs for 1-3 days. Data were expressed as the mean \pm SEM. 


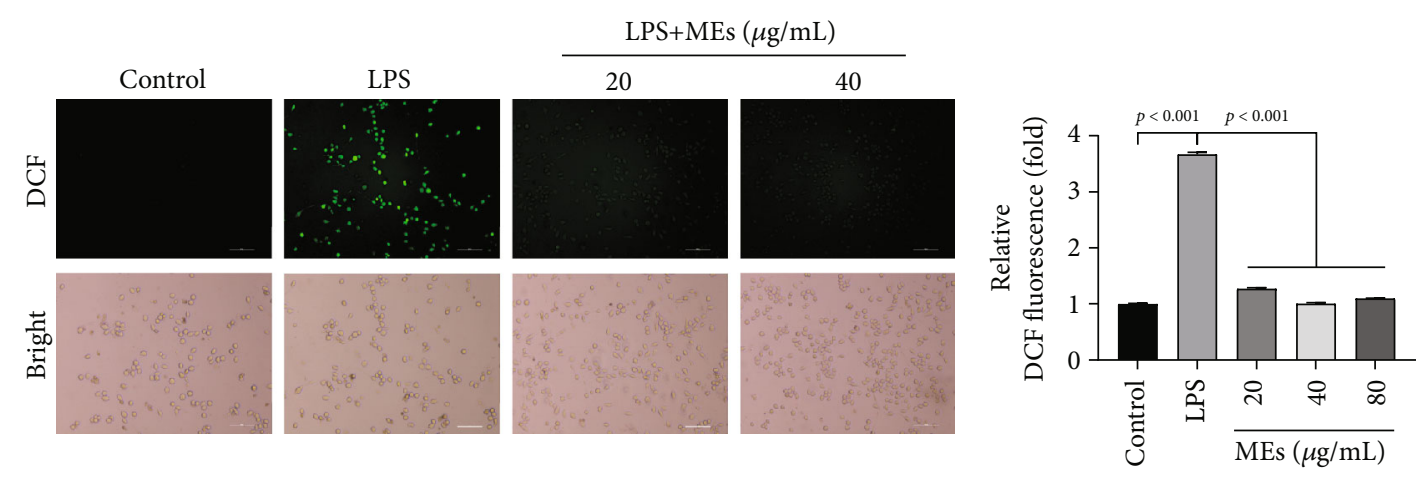

(a)
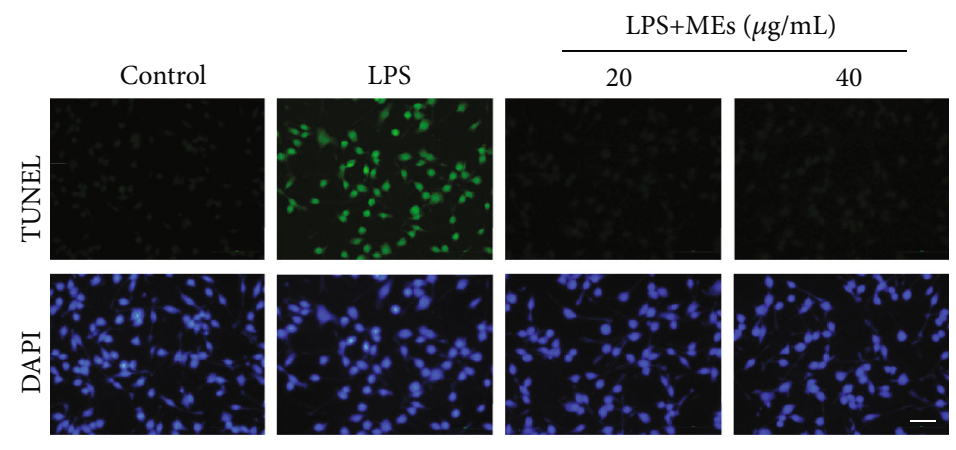

(b)

FIGURE 3: MEs inhibit ROS generation in the activated microglia. (a) The endpoint microscope fluorescence image of intracellular ROS of BV2 cells. Intracellular ROS generation was measured by fluorescence staining with $\mathrm{H}_{2}$ DCF-DA. LPS (200 ng/mL)-activated BV2 cells were treated with MEs $(20$ and $40 \mu \mathrm{g} / \mathrm{mL}$ ), scale bar $=100 \mu \mathrm{m}$. The quantitative analysis of the fluorescence intensity of DCF shown in (a). (b) TUNEL staining. LPS (200 ng/mL)-activated BV2 cells were treated with MEs (20 and $40 \mu \mathrm{g} / \mathrm{mL})$ for $24 \mathrm{~h}$. DNA fragmentation was detected by TUNEL assay. Scale bar $=100 \mu \mathrm{m}$.

BV2 cells caused an $\sim 40 \%$ decrease of SH-SY5Y cellular viability (Figure S3). MEs decreased the cellular toxicity of conditioned media from the LPS-treated BV2 cells in a concentration-dependent manner (Figure S3). These results show that MEs could protect DAergic neurons from microglia-mediated neurotoxicity.

The expression of LPS-induced ROS and proinflammatory factors is governed by the MAPKs and NF- $\kappa \mathrm{B}[33,34]$. In order to dissect how MEs downregulate the LPS-induced ROS and proinflammatory factors, the effect of MEs on MAPKs and NF- $\kappa$ B activities was measured. LPS stimulation significantly increased the phosphorylation of p65 subunit of NF- $\kappa \mathrm{B}, \mathrm{ERK} 1 / 2$, JNK, and p38 (Figures $4(\mathrm{e})$ and $4(\mathrm{f})$ ). TAK242, a classic TLR4 antagonist, was used here as a positive control. Similar to TAK242, MEs inhibited the LPSinduced phosphorylation of NF- $\kappa \mathrm{B}$ p 65 , ERK1/2, p38, and JNK in a concentration-dependent manner (Figures 4(e) and $4(\mathrm{f}))$.

Taken together, these results suggested that MEs suppressed the LPS-induced MAPK and NF- $\kappa \mathrm{B}$ activation, therefore inhibiting ROS and proinflammatory factors in the activated microglia and protecting DAergic neurons from the microglia-mediated neurotoxicity.

3.3. MEs Improve Parkinsonism in MPTP-Treated Mice. The etiology of PD indicates that ROS and proinflammatory factors $[14,28]$, particularly the generation of ROS, NO, and proinflammatory cytokines by activated microglia [35], mediates the majority of DAergic neuron destruction [20]. Cellular data shows that MEs of velvet antler inhibit ROS and neuroinflammation in the activated microglia and protect DAergic neurons from the microglia-mediated neurotoxicity. To confirm whether MEs could prevent and treat $\mathrm{PD}$, the effect of MEs on the MPTP-induced PD mouse model was investigated. It should be noted that MPTP is the gold standard for toxin-based PD animal models [30, $31,36]$, as it recapitulates the primary pathological and biochemical features of PD [37]. As shown in Figure 5(a), PD syndrome was induced by intraperitoneal administrations of MPTP, and MEs were administered once per day since the first MPTP injection for 5 days. Rotarod tests were performed $48 \mathrm{~h}$ after the final injection to determine whether MEs protected against the motor deficits caused by MPTP neurotoxicity. Compared to the vehicle control (918 $\mathrm{s} \pm 12 \mathrm{~s}$ ), the staying time of MPTP-treated mice on the rotating rod (145s $\pm 16 s$ ) was much shorter (Figure 5(b)). This is not surprising since subacute treatment of MPTP induces significant loss of neurons in the nigrostriatal pathway (Figure S4), which plays important roles in motor function [38]. ME treatment increased neurons in the substantia nigra of MPTP-treated mice (Figure S4) and substantially improved the performance of MPTP-treated mice to $776 \mathrm{~s} \pm 29 \mathrm{~s}$ (Figure 5(b)). These results suggest that MEs improve the behavioral deficits in MPTP-induced PD mice. 


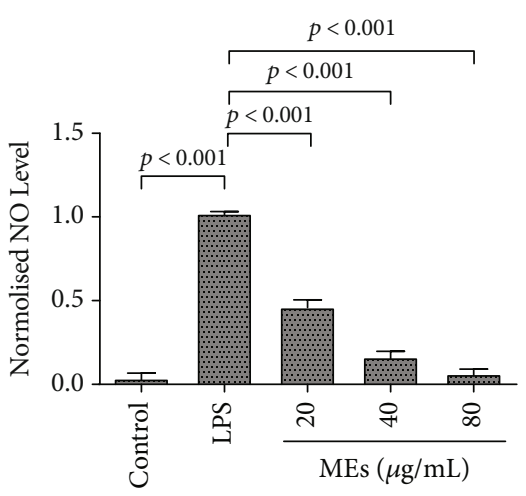

(a)

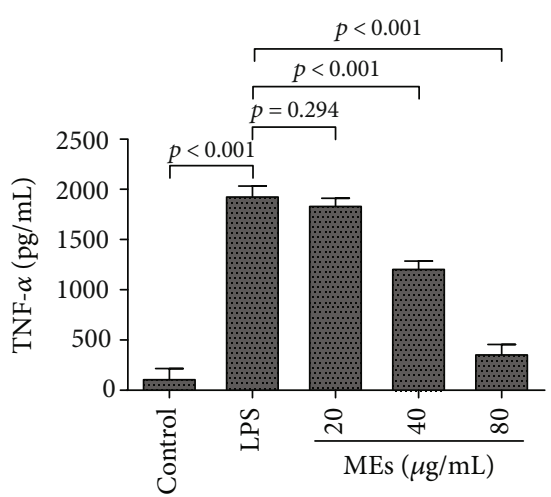

(b)

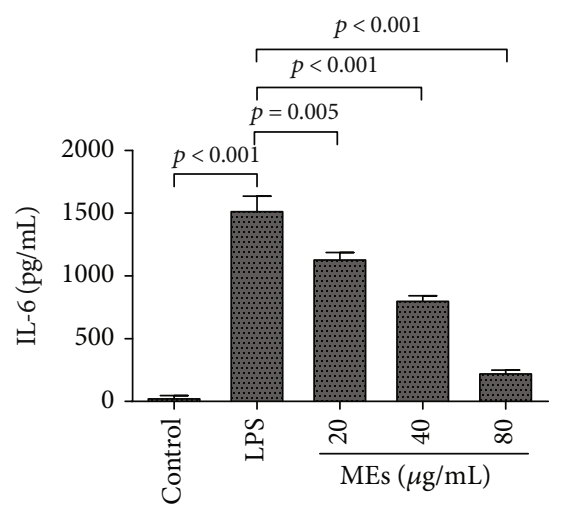

(c)
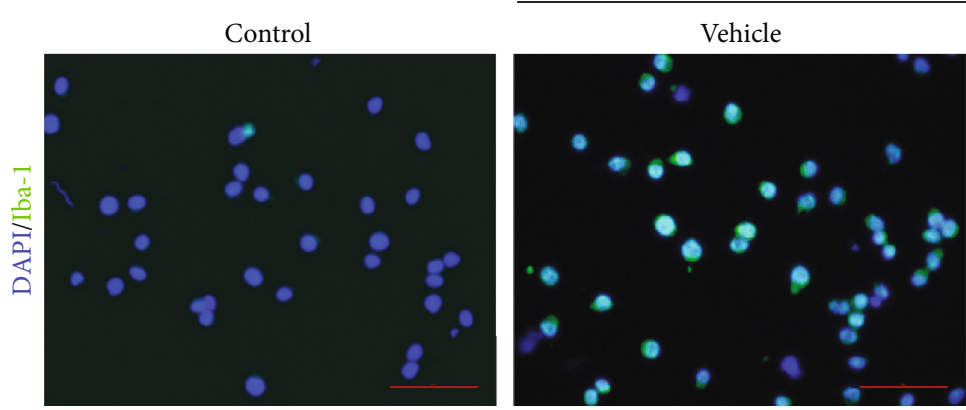

(d)

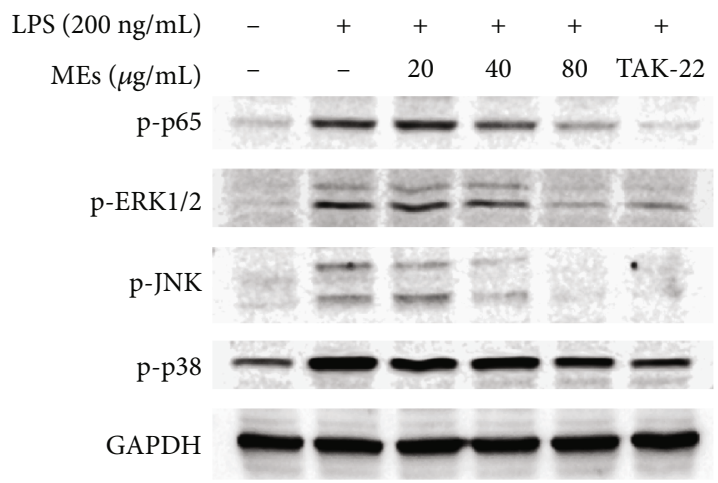

(e)

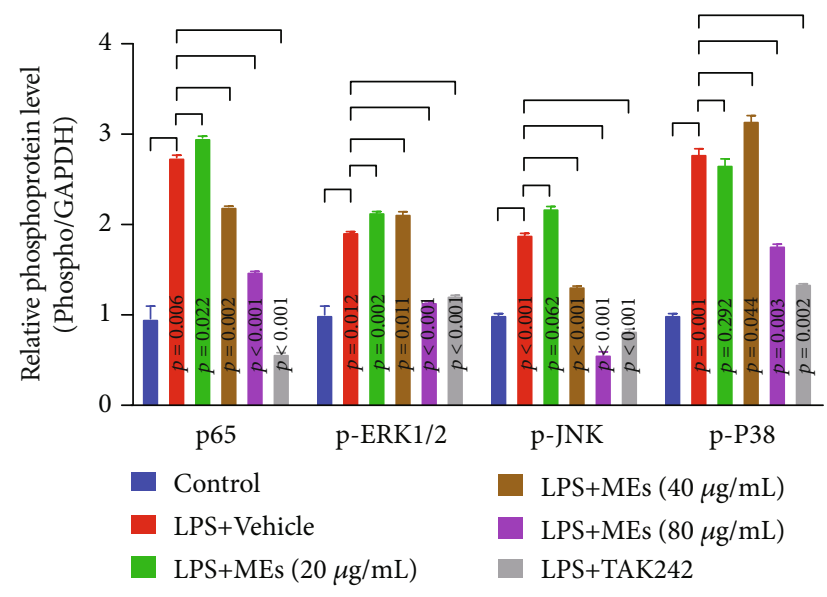

(f)

FIGURE 4: MEs inhibit proinflammatory factors by suppressing MAPKs and NF- $\kappa$ B activation in the activated microglia. (a-c) MEs inhibited LPS-induced NO (a), TNF- $\alpha$ (b), and IL-6 (c). BV2 cells were treated with LPS ( $200 \mathrm{ng} / \mathrm{mL}$ ) and indicated concentrations of MEs. After $24 \mathrm{~h}$ treatment, NO, TNF- $\alpha$, and IL-6 in the supernatant were measured; data are expressed as mean \pm SD. (d) The endpoint microscope immunofluorescence image of Iba-1 in BV2 cells. BV2 cells were treated with LPS $(200 \mathrm{ng} / \mathrm{mL})$ and indicated concentration of MEs for $24 \mathrm{~h}$. Iba-1 immunofluorescence staining was subsequently performed. Scale bar $=100 \mu \mathrm{m}$. (e, f) Effect of MEs on the LPS-induced phosphorylation of p65, ERK, JNK, and p38. BV2 cells were treated with LPS $(200 \mathrm{ng} / \mathrm{mL})$ and indicated concentrations of MEs for $1.5 \mathrm{~h}$. Cell lysates were prepared, and the protein samples were analyzed by western blot analysis. TAK-242 (1 $\mu \mathrm{M})$, a classic TLR4 antagonist, was used as the control. Results are representative of those obtained from three independent experiments.

$\mathrm{PD}$ is characterized by the degeneration of DAergic neuron in substantia nigra (SN), leading to a reduction of striatal dopamine [39]. Tyrosine hydroxylase (TH) catalyses the formation of L-dihydroxyphenylalanine (L-DOPA) [40], the rate-limiting step in the biosynthesis of dopamine [41]. There- fore, the TH level is closely associated with DAergic neuron function, and the reduction of the TH level in the brain tissues is a direct indication of DAergic neuron loss [42]. As shown in Figure 5(c), IHC analysis showed that MPTP-treated mice had decreased $\mathrm{TH}$-positive neurons in the $\mathrm{SN}$ and striatum 

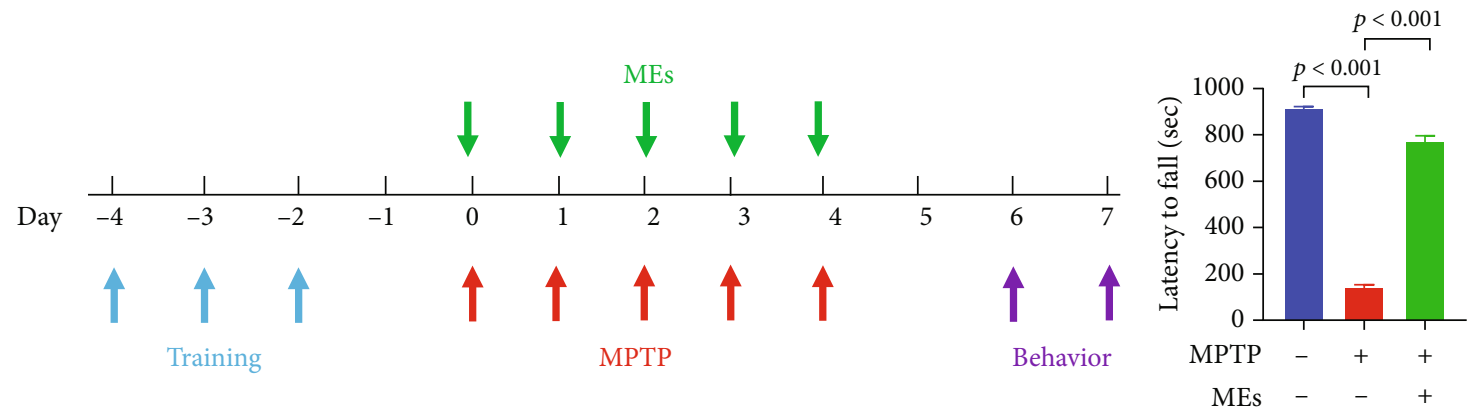

(a)

(b)
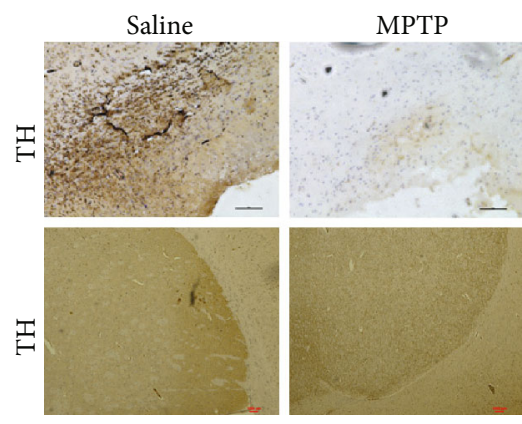

MEs
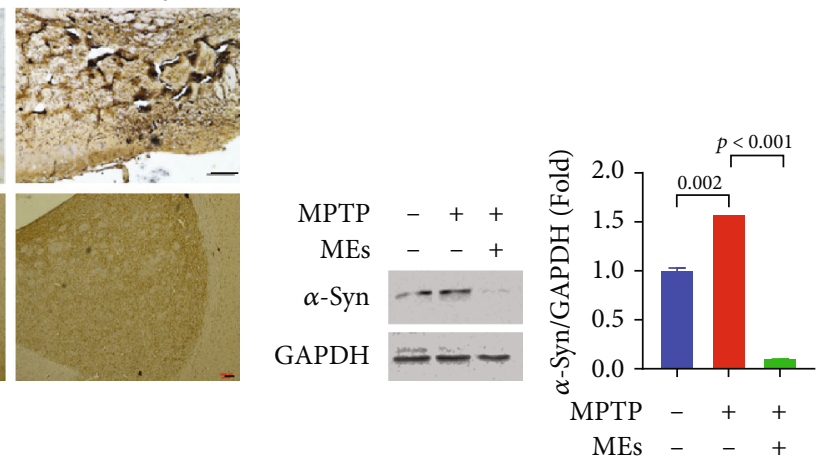

(c)

(d)

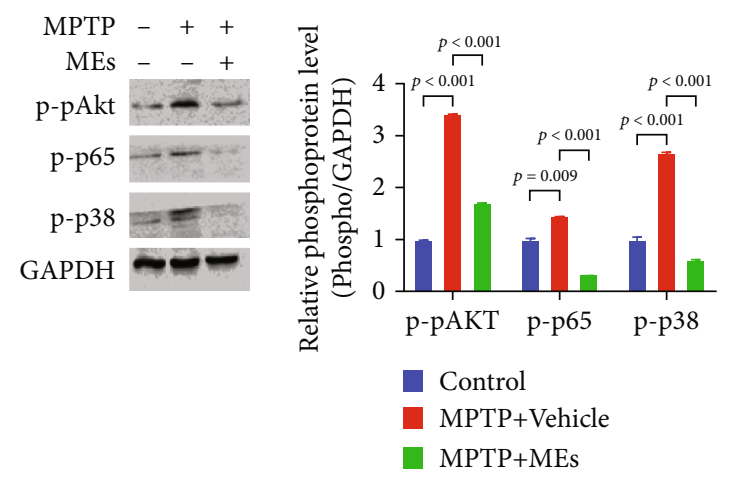

(e)

Figure 5: Continued. 

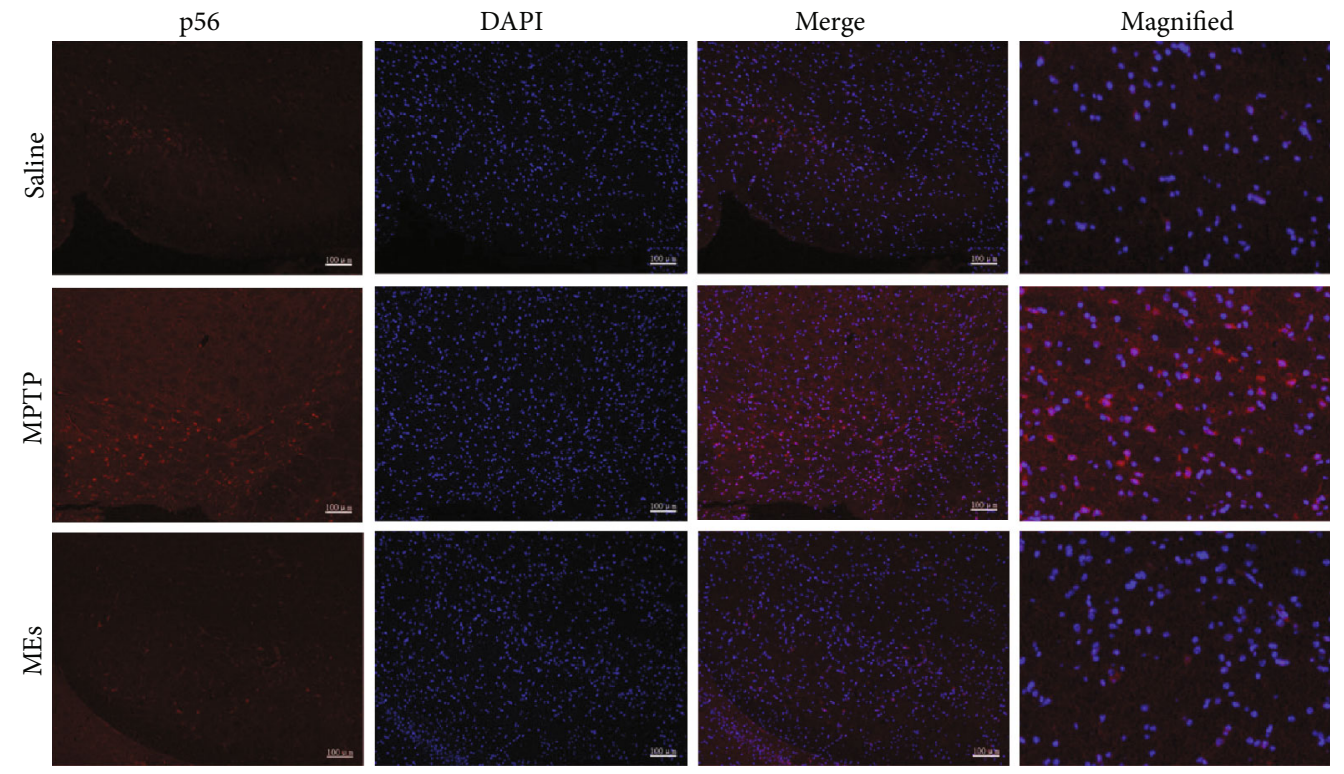

(f)

FIGURE 5: MEs improve parkinsonism in MPTP-treated mice. (a) Experimental timeline for the construction of the MPTP-induced PD mouse model and the administration of MEs. C57BL/6 mice (male, 7-8-week-old) were injected MPTP intraperitoneally at $30 \mathrm{mg} / \mathrm{kg} / \mathrm{day}$ for five days, and MEs (30 mg/kg/day) were injected intraperitoneally for 5 days since the administration of MPTP. On day 6, the rotarod test was performed. On day 7, mice were sacrificed and tissues were prepared for immunohistochemical (IHC) and western blotting. (b) Rotarod behavioral performance of MPTP-induced PD mice after ME treatment. Data are presented as the mean \pm SD $(n=6)$. (c) Immunohistochemistry for tyrosine hydroxylase $(\mathrm{TH})$ in the substantia nigra (scale bar $=100 \mu \mathrm{m})$ and striatum $(\mathrm{scale}$ bar $=1000 \mu \mathrm{m})$. $(\mathrm{d})$ Expression of $\alpha$-syn by western blot analysis. The blots were reprobed to detect GAPDH as the internal control. (e) Effect of MEs on the LPSinduced phosphorylation of Akt, p65, and p38 in the striatum. The protein in obtained tissue was analyzed and quantified by western blotting. (f) Effect of MEs on the nuclear translocation of NF- $\kappa \mathrm{B}$ in substantia nigra (scale bar $=100 \mu \mathrm{m}$ ). C57BL/6 mice (male, 7-8-week-old) were injected MPTP intraperitoneally at $30 \mathrm{mg} / \mathrm{kg} /$ day for five days, and MEs $(30 \mathrm{mg} / \mathrm{kg})$ were injected intraperitoneally for 5 days since the administration of MPTP. On day 7, mice were sacrificed and substantia nigra tissues were collected, and immunofluorescence was performed.

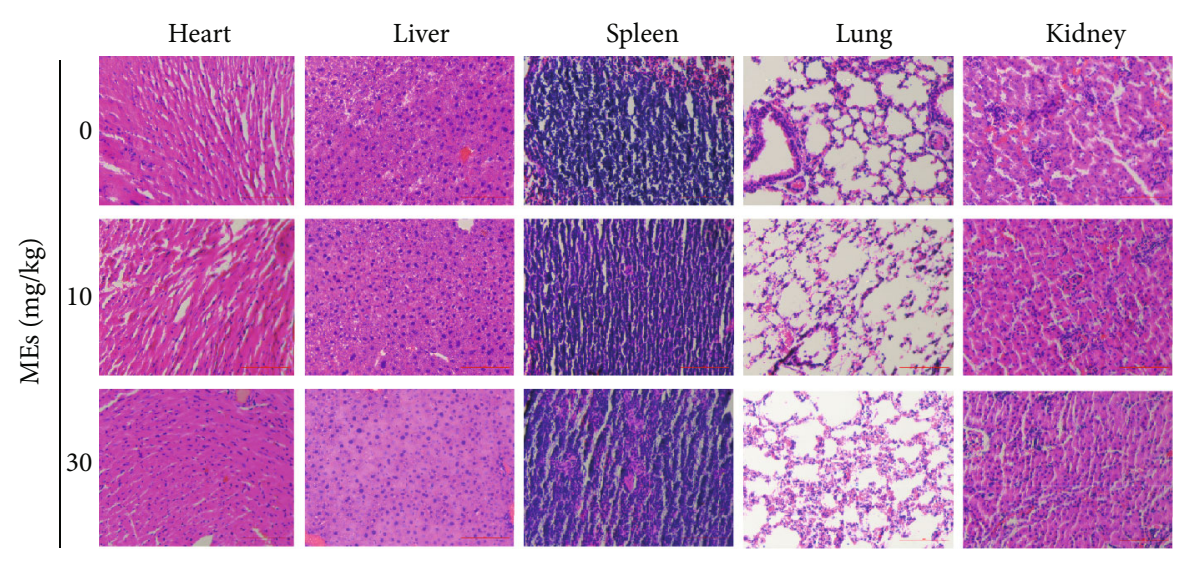

FIGURE 6: Acute toxicity of MEs in different organs. C57BL/6 mice (male, 7-8-week-old) were treated with indicated concentrations of MEs for 7 days. Mice were sacrificed, and different organs (heart, liver, spleen, lung, and kidney) were collected for hematoxylin and eosin (H\&E) staining. Scale bar $=100 \mu \mathrm{m}$.

compared to the vehicle control group. MEs significantly attenuated the MPTP-induced decrease of TH-positive neurons in the SN and striatum (Figure 5(c)). These results indicated that MEs could significantly reverse the decrease of $\mathrm{TH}$ expression and the loss of DAergic neurons in the MPTPinduced PD mice, which is consistent with the notion that increasing nigrostriatal $\mathrm{TH}$ expression is an effective therapeutic strategy for PD [43].

$\alpha$-Synuclein, a key protein critically involved in PD pathogenesis [44], is a major component of Lewy bodies [45], which are the neuropathological hallmarks of PD [46]. Aggregated $\alpha$-synuclein interacts with the cell membrane of 
neurons to form pore-like structures and depolarize the membrane potential, which disrupts the normal functions of the cell and leads to DAergic neuron death and progress of $\mathrm{PD}[47,48]$. Therefore, $\alpha$-synuclein is a therapeutic target for PD [49]. As shown in Figure 5(d), MPTP administration induced a significant upregulation of $\alpha$-synuclein protein in the striatum compared to the vehicle control, which is in agreement with the observed decrease of staying time of MPTP-treated mice on the rotating rod. Much less $\alpha$-synuclein protein expression was observed in the striatum of mice cotreated with MPTP and MEs (Figure 5(d)). These results indicate that MEs can protect PD development through downregulating $\alpha$-synuclein protein expression.

Akt, NF- $\kappa \mathrm{B}$, and p38 are dysregulated in the brain of PD patients [24, 50-52] and are the main components in the signal transduction pathway predominantly responsible for the generation of ROS and proinflammatory factors [53]. To investigate how MEs affect ROS and neuroinflammation in MPTP-induced PD mice, the effect of MEs on the phosphorylation of Akt, NF- $\kappa \mathrm{B}$, and $\mathrm{p} 38$ in the striatum was measured. As shown in Figure 5(e), MPTP treatment induced a significant upregulation of p-Akt, p-p65, and p-p38 in the striatum when compared to the vehicle control group. MEs substantially reduced the MPTPinduced Akt, NF- $\kappa$ B, and p38 activation (Figure 5(e)). In addition, NF- $\kappa \mathrm{B}$ nuclear translocation was found in MPTP-induced PD mice [30]; ME treatment could inhibit this nuclear translocation of $\mathrm{NF}-\kappa \mathrm{B}$ in our study (Figure 5(f)). In agreement with in vitro study, these in vivo results imply that MEs inhibit Akt, NF- $\kappa \mathrm{B}$, and p38.

Last, the acute toxicology of MEs was investigated. Hematoxylin and eosin- (H\&E-) staining of different organs (including the heart, liver, kidney, spleen, and lung) of mice treated with MEs for 7 days was examined (Figure 6). No apparent damage was detected in the examined organs, which indicates low safety concern of MEs. This is not surprising since velvet antler has been used as medicine and tonic food in East Asia for over two thousand years [4].

\section{Conclusions}

In all, this study dissected the pharmacology of MEs of velvet antler in PD. The C. elegans PD model studies show that MEs inhibit $\alpha$-synuclein aggregation and protect DAergic neurons from degeneration. In vitro microglia cellular data indicate that MEs suppressed the LPSinduced MAPK and NF- $\kappa \mathrm{B}$ activation, therefore inhibiting ROS and proinflammatory factors as well as the activation of microglia and protecting DAergic neurons from the microglia-mediated neurotoxicity. In vivo MPTP-induced PD mouse investigations demonstrate that MEs prevent MPTP-induced neuron loss in the substantia nigra and improve parkinsonism in MPTP-treated mice by increasing the expression level of $\mathrm{TH}$ and downregulating $\alpha$ synuclein protein expression. Since MEs of velvet antler have no apparent toxicology, MEs would have good translational potential for preventing and treating PD.

\section{Data Availability}

The accessibility data used to support the findings of this study were collected according to scientific research criteria and can be available from the corresponding authors upon request.

\section{Conflicts of Interest}

The authors declare that they have no conflicts of interest.

\section{Authors' Contributions}

Xiaohui Wang and Yinghua Peng conceived and designed the experiments. Ying Liu, Hongyuan Li, Min Yang, and Yunfei Li carried out the experimental work. Ying Liu and Hongyuan Li collected and processed the data. All the authors discussed the results, contributed to the final version of the manuscript, and approved the submitted version. All authors have read and agreed to the published version of the manuscript. Ying Liu and Hongyuan Li contributed equally to this work.

\section{Acknowledgments}

This work was supported by the National Key Research and Development Program of China (2018YFC1706603-06), Chinese Academy of Sciences (CAS) Pioneer Hundred Talents Program, Young Talents Program of Chinese Academy of Agricultural Sciences, grants from the Department of Science and Technology of Jilin Province (20180101021JC and 20180520059JH), and State Key Laboratory of Medicinal Chemical Biology, Nankai University (2018076).

\section{Supplementary Materials}

Figure S1: MEs had little damage to the DAergic neurons. The endpoint microscope fluorescence image of DAergic neurons in BZ555 worms treated with or without MEs o n days 1,2 , and 3 past the adult stage. The BZ555 worms synchronized at theL4 larval stage were not exposed to 6-OHDA and subsequently started to be exposed to $100 \mu \mathrm{g} / \mathrm{mL}$ MEs for 1-3 days. Figure S2: MEs had little cellular toxicity. BV2 cells were treated with different concentrations of MEs for $24 \mathrm{~h}$. Cellular viability was measured by MTT assay. Data are expressed as mean \pm SD, n.s., $p>0$.05. Figure S3: MEs protect DAergic neurons from microglia-mediated neurotoxicity. BV2 cells were treated with LPS $(200 \mathrm{ng} / \mathrm{mL})$ and indicated concentrations of MEs for $30 \mathrm{~h}$. The conditioned medium of microglial cells was then transferred to SH-SY5Y cell culture. After $24 \mathrm{~h}$ of incubation, the cell viability of SH-SY5Y cells was analyzed by MTT assay. Data are expressed as the mean \pm SD. Figure S4: ME treatment increased neurons in the substantia nigra of MPTP-treated mice. C57BL/6 mice (male, 7-8-week-old) were injected MPTP intraperitoneally at $30 \mathrm{mg} / \mathrm{kg} /$ day for five days, and MEs $(30 \mathrm{mg} / \mathrm{kg}$ ) were injected intraperitoneally for 5 days since the administration of MPTP. On day 7, mice were sacrificed, and substantia nigra tissues were collected, and hematoxylin and eosin (H\&E) staining was performed. Scale bar $=100 \mu \mathrm{m}$, right part (40x magnification). (Supplementary materials) 


\section{References}

[1] S. M. Hague, S. Klaffke, and O. Bandmann, "Neurodegenerative disorders: Parkinson's disease and Huntington's disease," Journal of Neurology, Neurosurgery \& Psychiatry, vol. 76, no. 8, pp. 1058-1063, 2005.

[2] J. M. Beitz, "Parkinson s disease a review," Frontiers in Bioscience, vol. S6, no. 1, pp. 65-74, 2014.

[3] A. Vallee, Y. Lecarpentier, R. Guillevin, and J. N. Vallee, "Circadian rhythms, neuroinflammation and oxidative stress in the story of Parkinson's disease," Cells, vol. 9, no. 2, p. 314, 2020.

[4] Z. Sui, L. Zhang, Y. Huo, and Y. Zhang, "Bioactive components of velvet antlers and their pharmacological properties," Journal of Pharmaceutical and Biomedical Analysis, vol. 87, pp. 229240, 2014.

[5] S. H. Lee, H. W. Yang, Y. Ding et al., "Anti-inflammatory effects of enzymatic hydrolysates of velvet antler in Raw 264.7 cells in vitro and zebrafish model," EXCLI Journal, vol. 14, pp. 1122-1132, 2015.

[6] Z. J. Zang, H. F. Tang, Y. Tuo et al., "Effects of velvet antler polypeptide on sexual behavior and testosterone synthesis in aging male mice," Asian Journal of Andrology, vol. 18, no. 4, pp. 613-619, 2016.

[7] X. Wang, H. Li, Y. Liu et al., "Velvet antler methanol extracts (MEs) protects against oxidative stress in Caenorhabditis elegans by SKN-1," Biomedicine \& Pharmacotherapy, vol. 121, p. 109668, 2020.

[8] L. You, J. Wang, T. Liu et al., "Targeted brain delivery of rabies virus glycoprotein 29-modified deferoxamine-loaded nanoparticles reverses functional deficits in parkinsonian mice," ACS Nano, vol. 12, no. 5, pp. 4123-4139, 2018.

[9] J. F. Cooper and J. M. Van Raamsdonk, "Modeling Parkinson's disease in C. elegans," Journal of Parkinson's Disease, vol. 8, no. 1, pp. 17-32, 2018.

[10] The C elegans Sequencing Consortium, "Genome sequence of the nematode C.\&nbsp;elegans: a platform for investigating biology," Science, vol. 282, no. 5396, pp. 2012-2018, 1998.

[11] O. Hobert, "PCR fusion-based approach to create reporter gene constructs for expression analysis in transgenic C-elegans," BioTechniques, vol. 32, no. 4, pp. 728-730, 2002.

[12] K. Kawamura and I. N. Maruyama, "Forward genetic screen for Caenorhabditis elegans mutants with a shortened locomotor healthspan," G3-Genes Genomes Genetics, vol. 9, no. 8, pp. 2415-2423, 2019.

[13] S. Honnen, "Caenorhabditis elegans as a powerful alternative model organism to promote research in genetic toxicology and biomedicine," Archives of Toxicology, vol. 91, no. 5, pp. 2029-2044, 2017.

[14] V. Dias, E. Junn, and M. M. Mouradian, "The role of oxidative stress in Parkinson's disease," Journal of Parkinson's Disease, vol. 3, no. 4, pp. 461-491, 2013.

[15] Z. D. Zhou, S. P. Xie, W. T. Saw et al., "The therapeutic implications of tea polyphenols against dopamine (DA) neuron degeneration in Parkinson's disease (PD)," Cells, vol. 8, no. 8, p. 911, 2019.

[16] F. E. Frerman, "Reaction of electron-transfer flavoprotein ubiquinone oxidoreductase with the mitochondrial respiratorychain," Biochimica et Biophysica Acta, vol. 893, no. 2, pp. 161-169, 1987.

[17] R. A. Gregg, M. H. Baumann, J. S. Partilla et al., "Stereochemistry of mephedrone neuropharmacology: enantiomer-specific behavioural and neurochemical effects in rats," British Journal of Pharmacology, vol. 172, no. 3, pp. 883-894, 2015.

[18] D. L. Chase and M. R. Koelle, "Biogenic amine neurotransmitters in C. elegans," WormBook, pp. 1-15, 2007.

[19] M. P. Smith and W. A. Cass, "Oxidative stress and dopamine depletion in an intrastriatal 6-hydroxydopamine model of Parkinson's disease," Neuroscience, vol. 144, no. 3, pp. 1057-1066, 2007.

[20] E. Lazdon, N. Stolero, and D. Frenkel, "Microglia and Parkinson's disease: footprints to pathology," Journal of Neural Transmission, vol. 127, no. 2, pp. 149-158, 2020.

[21] C. Colton, S. Wilt, D. Gilbert, O. Chernyshev, J. Snell, and M. Dubois-Dalcq, "Species differences in the generation of reactive oxygen species by microglia," Molecular and Chemical Neuropathology, vol. 28, no. 1-3, pp. 15-20, 1996.

[22] A. Henn, S. Lund, M. Hedtjarn, A. Schrattenholz, P. Porzgen, and M. Leist, "The suitability of BV2 cells as alternative model system for primary microglia cultures or for animal experiments examining brain inflammation," ALTEX, vol. 26, no. 2, pp. 83-94, 2009.

[23] G. Dutta, P. Zhang, and B. Liu, "The lipopolysaccharide Parkinson's disease animal model: mechanistic studies and drug discovery," Fundamental and Clinical Pharmacology, vol. 22, no. 5, pp. 453-464, 2008.

[24] S. S. Singh, S. N. Rai, H. Birla, W. Zahra, A. S. Rathore, and S. P. Singh, "NF- $\kappa$ B-mediated neuroinflammation in Parkinson's disease and potential therapeutic effect of polyphenols," Neurotoxicity Research, vol. 37, no. 3, pp. 491-507, 2020.

[25] S. K. Yadav, S. N. Rai, and S. P. Singh, "Mucuna pruriens reduces inducible nitric oxide synthase expression in Parkinsonian mice model," Journal of Chemical Neuroanatomy, vol. 80, pp. 1-10, 2017.

[26] R.-O. Gheorghe, A. Deftu, A. Filippi et al., "Silencing the cytoskeleton protein Iba1 (ionized calcium binding adapter protein 1) interferes with BV2 microglia functioning," Cellular and Molecular Neurobiology, vol. 40, no. 6, pp. 1011-1027, 2020.

[27] D. Ito, Y. Imai, K. Ohsawa, K. Nakajima, Y. Fukuuchi, and S. Kohsaka, "Microglia-specific localisation of a novel calcium binding protein, Iba1," Brain Research. Molecular Brain Research, vol. 57, no. 1, pp. 1-9, 1998.

[28] R. L. Mosley, E. J. Benner, I. Kadiu et al., "Neuroinflammation, oxidative stress, and the pathogenesis of Parkinson's disease," Clinical Neuroscience Research, vol. 6, no. 5, pp. 261-281, 2006.

[29] J. A. Klein and S. L. Ackerman, "Oxidative stress, cell cycle, and neurodegeneration," Journal of Clinical Investigation, vol. 111, no. 6, pp. 785-793, 2003.

[30] S. N. Rai, H. Birla, S. S. Singh et al., "Mucuna pruriens protects against MPTP intoxicated neuroinflammation in Parkinson's disease through NF- $\kappa \mathrm{B} / \mathrm{pAKT}$ signaling pathways," Frontiers in Aging Neuroscience, vol. 9, p. 421, 2017.

[31] H. Birla, S. N. Rai, S. S. Singh et al., "Tinospora cordifolia suppresses neuroinflammation in Parkinsonian mouse model," NeuroMolecular Medicine, vol. 21, no. 1, pp. 42-53, 2019.

[32] H. Xie, H. Hu, M. Chang et al., "Identification of chaperones in a MPP ${ }^{+}$-induced and ATRA/TPA-differentiated SH-SY5Y cell PD model," American Journal of Translational Research, vol. 8, no. 12, pp. 5659-5671, 2016.

[33] N. Somensi, T. K. Rabelo, A. G. Guimarães et al., "Carvacrol suppresses LPS-induced pro-inflammatory activation in RAW 264.7 macrophages through ERK1/2 and NF-kB pathway," International Immunopharmacology, vol. 75, p. 105743, 2019. 
[34] N. Shang and J. Wu, "Egg-derived tripeptide IRW attenuates LPS-induced osteoclastogenesis in RAW 264.7 macrophages via inhibition of inflammatory responses and NF- $\kappa \mathrm{B} / \mathrm{MAPK}$ activation," Journal of Agricultural and Food Chemistry, vol. 68 , no. 22, pp. 6132-6141, 2020.

[35] M. E. Lull and M. L. Block, "Microglial activation and chronic neurodegeneration," Neurotherapeutics, vol. 7, no. 4, pp. 354$365,2010$.

[36] K. Renkawek, "Experimental model of Parkinson disease induced by N-methyl-4-phenyl-1,2,3,6-tetrahydropyridine (MPTP)," Neuropatologia Polska, vol. 24, no. 1, pp. 1-8, 1986.

[37] M. R. Luquin, J. A. Obeso, M. T. Herrero, J. Laguna, and J. M. Martinez-Lage, "Parkinsonism induced by MPTP as an experimental model of Parkinson disease: similarities and differences," Neurología, vol. 6, no. 8, pp. 287-294, 1991.

[38] M. G. Jo, M. Ikram, M. H. Jo et al., "Gintonin mitigates MPTPinduced loss of nigrostriatal dopaminergic neurons and accumulation of $\alpha$-synuclein via the Nrf2/HO-1 pathway," Molecular Neurobiology, vol. 56, no. 1, pp. 39-55, 2019.

[39] F. Fornai, A. di Poggio, A. Pellegrini, S. Ruggieri, and A. Paparelli, "Noradrenaline in Parkinson's disease: from disease progression to current therapeutics," Current Medicinal Chemistry, vol. 14, no. 22, pp. 2330-2334, 2007.

[40] J. M. King, G. Muthian, V. Mackey, M. Smith, and C. Charlton, "L-Dihydroxyphenylalanine modulates the steady-state expression of mouse striatal tyrosine hydroxylase, aromatic L-amino acid decarboxylase, dopamine and its metabolites in an MPTP mouse model of Parkinson's disease," Life Sciences, vol. 89, no. 17-18, pp. 638-643, 2011.

[41] K. M. Bowling, Z. Huang, D. Xu et al., "Direct binding of GTP cyclohydrolase and tyrosine hydroxylase," Journal of Biological Chemistry, vol. 283, no. 46, pp. 31449-31459, 2008.

[42] G. Alam, M. Edler, S. Burchfield, and J. R. Richardson, "Single low doses of MPTP decrease tyrosine hydroxylase expression in the absence of overt neuron loss," Neurotoxicology, vol. 60, pp. 99-106, 2017.

[43] A. Iarkov, G. E. Barreto, J. A. Grizzell, and V. Echeverria, "Strategies for the treatment of Parkinson's disease: beyond dopamine," Frontiers in Aging Neuroscience, vol. 12, 2020.

[44] S. Gebel, A. Ashrafi, P. Garcia et al., "Gene expression analysis using the Parkinson's disease map reveals early effects of alpha-synuclein on PD pathogenesis," Movement Disorders, vol. 31, pp. S233-S233, 2016.

[45] D. J. Koss, D. Erskine, J. Attems, and T. Outeiro, "Resolving the issue of nuclear localisation of alpha-synuclein in Lewy body diseases," Neuropathology and Applied Neurobiology, vol. 46, pp. 21-21, 2020.

[46] H. Geut, D. H. Hepp, E. Foncke et al., "Neuropathological correlates of parkinsonian disorders in a large Dutch autopsy series," Acta Neuropathologica Communications, vol. 8, no. 1, p. 39, 2020.

[47] J. Prakash, S. Chouhan, S. K. Yadav, S. Westfall, S. N. Rai, and S. P. Singh, "Withania somnifera alleviates parkinsonian phenotypes by inhibiting apoptotic pathways in dopaminergic neurons," Neurochemical Research, vol. 39, no. 12, pp. 25272536, 2014.

[48] N. M. Bonini and B. I. Giasson, "Snaring the function of $\alpha$-synuclein," Cell, vol. 123, no. 3, pp. 359-361, 2005.

[49] L. Kalia, "Alpha-synuclein as a potential biomarker and therapeutic target for Parkinson's disease," Journal of Neurochemistry, vol. 150, pp. 35-35, 2019.
[50] S. Timmons, M. F. Coakley, A. M. Moloney, and C. O'Neill, "Akt signal transduction dysfunction in Parkinson's disease," Neuroscience Letters, vol. 467, no. 1, pp. 30-35, 2009.

[51] J. He, W. Zhong, M. Zhang, R. Zhang, and W. Hu, "P38 mitogen-activated protein kinase and Parkinson's disease," Translational Neuroscience, vol. 9, no. 1, pp. 147-153, 2018.

[52] A. Bellucci, L. Bubacco, F. Longhena et al., "Nuclear factor- $\kappa$ B dysregulation and $\alpha$-synuclein pathology: critical interplay in the pathogenesis of Parkinson's disease," Frontiers in Aging Neuroscience, vol. 12, p. 68, 2020.

[53] S. K. Jha, N. K. Jha, R. Kar, R. K. Ambasta, and P. Kumar, "p38 MAPK and PI3K/AKT signalling cascades inParkinson's disease," International Journal of Molecular Medicine, vol. 4, no. 2, pp. 67-86, 2015. 